\title{
Stability of rigid rotors supported by air foil bearings: Comparison of two fundamental
} approaches

\author{
Larsen, Jon Steffen; Santos, IImar; von Osmanski, Alexander Sebastian
}

Published in:

Journal of Sound and Vibration

Link to article, DOI:

10.1016/j.jsv.2016.06.022

Publication date:

2016

Document Version

Peer reviewed version

Link back to DTU Orbit

Citation (APA):

Larsen, J. S., Santos, I., \& von Osmanski, A. S. (2016). Stability of rigid rotors supported by air foil bearings:

Comparison of two fundamental approaches. Journal of Sound and Vibration, 381, 179-191.

https://doi.org/10.1016/j.jsv.2016.06.022

\section{General rights}

Copyright and moral rights for the publications made accessible in the public portal are retained by the authors and/or other copyright owners and it is a condition of accessing publications that users recognise and abide by the legal requirements associated with these rights.

- Users may download and print one copy of any publication from the public portal for the purpose of private study or research.

- You may not further distribute the material or use it for any profit-making activity or commercial gain

- You may freely distribute the URL identifying the publication in the public portal 


\title{
Stability of rigid rotors supported by air foil bearings: Comparison of two fundamental approaches
}

\author{
Jon S. Larsen ${ }^{\text {a }}$, Ilmar F. Santos ${ }^{\text {a }}$, Sebastian von Osmanski ${ }^{\text {a }}$ \\ ${ }^{a}$ Department of Mechanical Engineering, Technical University of Denmark, 2800 Kgs. Lyngby, Denmark
}

\begin{abstract}
High speed direct drive motors enable the use of Air Foil Bearings (AFB) in a wide range of applications due to the elimination of gear forces. Unfortunately, AFB supported rotors are lightly damped, and an accurate prediction of their Onset Speed of Instability (OSI) is therefore important. This paper compares two fundamental methods for predicting the OSI. One is based on a nonlinear time domain simulation and another is based on a linearised frequency domain method and a perturbation of the Reynolds equation. Both methods are based on equivalent models and should predict similar results. Significant discrepancies are observed leading to the question, is the classical frequency domain method sufficiently accurate? The discrepancies and possible explanations are discussed in detail.
\end{abstract}

Keywords:

Air foil bearing, rigid rotor, transient simulation, nonlinear analysis, stability

\section{Nomenclature}

CG Center of Gravity

FE Finite Element

LD Logarithmic Decrement, $\operatorname{LD}=-2 \pi \operatorname{Re}\left(\lambda_{i}\right) / \operatorname{Im}\left(\lambda_{i}\right)$

ODE Ordinary Differential Equation

OSI Onset Speed of Instability

PDE Partial Differential Equation

(*) Time derivative, $d^{2} / d \tau^{2}$

() Time derivative, $d / d \tau$

$\nabla$. Divergence

$\nabla \quad$ Gradient, $\nabla=\{\partial / \partial \theta, \partial / \partial \tilde{z}\}$

$A, B \quad$ Bearings

$C \quad$ Radial clearance

$D, \tilde{D} \quad$ Bearing damping, $\tilde{D}=\omega C /\left(p_{a} R^{2}\right) D$

$E \quad$ Modulus of elasticity of foil

$F, \tilde{F} \quad$ Bearing force components, $\tilde{F}=1 /\left(p_{a} R^{2}\right) F$

$I \quad$ Mass moment of inertia

$K, \tilde{K} \quad$ Bearing stiffness, $\tilde{K}=C /\left(p_{a} R^{2}\right) K$

$L, \tilde{L} \quad$ Bearing length, $\tilde{L}=L / R$

$N \quad$ Number of fluid film nodes

$N_{p} \quad$ Number of pads

$R \quad$ Journal radius

$S \quad$ Compressibility number, $S=6 \mu \omega / p_{a}(R / C)^{2}$
$S_{b} \quad$ Bump foil pitch

$W, \tilde{W} \quad$ Static load components, $\tilde{W}=1 /\left(p_{a} R^{2}\right) W$

$a \quad$ Scaler field quantity

$b, \tilde{b} \quad$ Structural damping per unit area,

$\tilde{b}=C /\left(p_{a} \omega\right) b$

$e, \varepsilon \quad$ Journal eccentricity (or $e=$ element), $\varepsilon=e / C$

$f_{\gamma} \quad$ Direction cosines

$h, \tilde{h} \quad$ Film height, $\tilde{h}=h / C$

$h_{b} \quad$ Bump foil height

$h_{c}, \tilde{h}_{c} \quad$ Film height correction, $\tilde{h}_{c}=h_{c} / C$

$h_{r}, \tilde{h}_{r} \quad$ Film height (rigid), $\tilde{h}_{r}=h_{r} / C$

$h_{s}, \tilde{h}_{s} \quad$ Slope height, $\tilde{h}_{s}=h_{s} / C$

$i \quad$ Pad, $i=1,2, \ldots, N_{p}$

$k, \tilde{k} \quad$ Structural stiffness per unit area, $\tilde{k}=C / p_{a} k$

$l_{0} \quad$ Bump half length

$l_{1}, l_{2} \quad$ Distance to bearings

$m \quad$ Mass

$p, \tilde{p} \quad$ Film pressure, $\tilde{p}=p / p_{a}$

$p_{a} \quad$ Ambient pressure

$p_{\gamma}, \tilde{p}_{\gamma} \quad$ Perturbed pressure, $\tilde{p}_{\gamma}=C p_{\gamma} / p_{a}$

$q, \tilde{q} \quad$ Structural flexibility per unit area, $\tilde{q}=p_{a} q / C=1 / \tilde{k}$

Email addresses: josla@mek.dtu.dk (Jon S. Larsen), ifs@mek.dtu.dk (Ilmar F. Santos), sebastian@osmanski.dk (Sebastian von Osmanski) 
$x, y, z, \tilde{z}$ Cartesian coordinates, $\tilde{z}=z / R$

$\Delta \varepsilon \quad$ Perturbation amplitude

$\Phi \quad$ Fluid domain

$\alpha \quad$ Bearing position

$\eta \quad$ Structural loss factor of foils

$\gamma \quad$ Coordinates $x, y$

$\lambda \quad$ Complex eigenvalue, $\lambda=\tilde{\omega}_{n} i+\tilde{\beta}$

$\mu \quad$ Dynamic viscosity

$\mu_{f} \quad$ Foil friction coefficient

$v \quad$ Poisson's ratio of foil

$\omega \quad$ Angular speed of journal

$\omega_{n}, \tilde{\omega}_{n} \quad$ Eigenfrequency, $\tilde{\omega}_{n}=\omega_{n} / \omega$

$\omega_{s}, \tilde{\omega}_{s}$ Excitation frequency, $\tilde{\omega}_{s}=\omega_{s} / \omega$

$\psi \quad$ Film state variable, $\psi=p h$

$\tau \quad$ Dimensionless time, $\tau=\omega t$

$\theta, \tilde{\theta} \quad$ Circumferential angle, $\tilde{\theta}=\theta R$

$\theta_{l} \quad$ First pad leading edge angle

$\theta_{s} \quad$ First pad slope extend

$\theta_{t} \quad$ First pad trailing edge angle

$\xi_{i}, \eta_{j} \quad$ Gauss points

$\mathbf{f}, \tilde{\mathbf{f}} \quad$ Bearing force vector, $\tilde{\mathbf{f}}=1 /\left(p_{a} R^{2}\right) \mathbf{f}$ r Residual vector

s Advection vector, $\mathbf{s}=\{S, 0\}^{T}$

$\mathbf{w}, \tilde{\mathbf{w}} \quad$ Load vector, $\tilde{\mathbf{w}}=1 /\left(p_{a} R^{2}\right) \mathbf{w}$

$\psi \quad$ Film state vector

$\tilde{\mathbf{h}}_{c} \quad$ Foil deformation vector

$\tilde{\mathbf{p}} \quad$ Pressure vector

$\tilde{\mathbf{p}}_{m} \quad$ Arithmetic mean pressure vector

$\boldsymbol{\varepsilon} \quad$ Eccentricity vector

a Nodal vector

g() Nonlinear vector function

y State vector

$\mathbf{z} \quad$ Rotor state vector

$\mathbf{z}_{1} \quad$ Rotor displacement vector, $\mathbf{z}_{1}=\boldsymbol{\varepsilon}$

$\mathbf{z}_{2} \quad$ Rotor velocity vector, $\mathbf{z}_{2}=\dot{\boldsymbol{\varepsilon}}$

0 Zero matrix

A State space matrix

B Shape function derivatives matrix

$\mathbf{D}, \tilde{\mathbf{D}} \quad$ Damping matrix, $\tilde{\mathbf{D}}=\omega C /\left(p_{a} R^{2}\right) \mathbf{D}$

$\mathbf{G}, \tilde{\mathbf{G}} \quad$ Gyroscopic matrix, $\tilde{\mathbf{G}}=\omega^{2} C /\left(p_{a} R^{2}\right) \mathbf{G}$

I Identity matrix

$\mathbf{K}, \tilde{\mathbf{K}} \quad$ Stiffness matrix, $\tilde{\mathbf{K}}=C /\left(p_{a} R^{2}\right) \mathbf{K}$

$\mathbf{M}, \tilde{\mathbf{M}} \quad$ Mass matrix, $\tilde{\mathbf{M}}=\omega^{2} C /\left(p_{a} R^{2}\right) \mathbf{M}$

$\mathbf{N} \quad$ Shape function matrix

$\boldsymbol{\Gamma} \quad$ Fluidity matrix

$\tilde{\mathbf{D}}_{s} \quad$ First order coefficients, $\tilde{\mathbf{D}}_{s}=\tilde{\mathbf{D}}-\tilde{\mathbf{G}}$

\section{Introduction}

For almost 150 years, bearing-influenced rotor vibrations have attracted the attention of engineers and researchers. In one of the first documents found in the literature, dated 1869, Rankine [1] reported the amplification of shaft lateral vibrations at different (critical) speeds. In 1894, Dunkerley [2] presented an approximate method for finding the natural frequencies or critical whirling speeds of simplified rotor systems. In 1919, Jeffcott [3] published one of the most significant contributions to rotor dynamics, in which the lateral vibration of loaded shafts in the neighbourhood of a whirling speed was addressed. With the significant developments of rotating machines in the 1920s, especially driven by steam turbines [4], the influence of fluid film bearings on the lateral shaft vibrations became a very important research topic. Motivated by the necessity of enlarging the operational range of steam turbines, the influence of higher angular velocities on the shaft lateral vibrations was investigated by Newkirk and Taylor [5] in 1925. They documented not only the increase of shaft lateral vibrations while crossing over the first critical speed but also, for the first time, the severe unexpected vibrations about twice the first critical speed. In 1927, with an iterative approach based on assumed shaft mode shapes and the new concept of "nonlinear oil springs", Stodola [6] linked rotor lateral vibrations, bearing stiffness and their dependence on journal eccentricity. Using a rigid rotor, Newkirk [7] experimentally proved in 1931 that half-speed whirl could occur over a wide speed range, well below twice the first critical speed. Using a simplified infinite-width bearing model and disregarding cavitation, Swift [8] analytically investigated the nonsteady conditions in journal bearings. Taking into account bearing as well as rotor flexibility, Smith [9] reported the possibility of four critical speeds for non-symmetrical rotors, a remarkable achievement in the beginning of the 1930s, an era characterised by the absence of digital computer power.

The development of digital computers since the 1940s has strongly facilitated researchers to evaluate more complex and sophisticated mathematical models for describing rotor and bearing dynamics. It has encouraged the development of new alternative procedures to predict bearing-influenced rotor dynamic response and stability [10-13] in the 1950s and 1960s. From the viewpoint of solid mechanics, rotors with complex geometry could be refined using the transfer matrix method $[14,15]$ and later on the finite element (FE) method [16] to account for distributed rotor 
mass, inertia, and gyroscopic effect. From the viewpoint of fluid film bearing dynamics, effects of fluid compressibility [17-19] and inertia [20-22], cavitation [23] and turbulence [24] could be numerically investigated and more accurately predicted. For example, lubricant compressibility strongly influences the static and dynamic behaviour of gas bearings as already reported by Harrison in 1913 [17]. With the advent of digital computers, gas bearings have been intensively investigated over the last five decades [18, 19, 25-35]. Even though the available computing power has notably increased, the numerical simulation of complex mathematical models - linking several physical domains in rotor-bearing dynamics - has become even more demanding. Such models arise, for example, when bump foil structural compliance, dry friction and gas compressibility are considered in the context of bump foil gas bearings.

In order to predict lateral vibration response and stability of rotors supported by bump foil gas bearings, linear [31-33, 35-42] and nonlinear analyses [34, 43-46] can be carried out. In the linear analysis, the gas film forces are represented by equivalent springs and dampers. The springs and dampers are represented by eight coefficients, strongly dependent on rotor-bearing system operational conditions and frequency of external excitation forces. Such force coefficients are calculated numerically or analytically, as proposed by Lund in 1968 [28] for gas bearings. One of the first theoretical contributions linking Lund's perturbation approach to obtain force coefficients of bump foil gas bearings is dated 1993 [40]. Such force coefficients are normally used to predict stability as well as unbalance response. Following such an approach other authors also investigated the stability of rotors supported by bump foil gas bearings [31, 32, 35, 36, 47].

In this paper, two fundamental approaches to predict lateral stability of rotor-bearing systems are presented and compared: one based on linearised force coefficients as introduced in 1993 [40] based on Lund's perturbation approach and currently used by several authors; another based on the nonlinear behaviour of the fluid film forces and time integration. A similar comparison by Hoffmann et. al. [47] has previously found good agreement between the methods, while another by Kim [31] reported significant discrepancies. In the latter, it should however be noted that very low stability limits were predicted by the applied linear approach.

The nonlinear time domain integration scheme applied in the present paper is based on a simultaneous solution of all state variables in time as originally proposed by Bonello and Pham $[43,46]$. This maintains the true simultaneously coupled nature of the equation system and hence alleviates the potential issues of temporal convergence suffered by decoupled integration schemes. Despite the larger time increments permitted by this simultaneous approach, the method of nonlinear time domain integration is not so frequently used due to the computational time demanded. This obstacle is partly handled in the present work using a highly optimised computer implementation, but it has been demonstrated in $[43,44]$ that the stability of a static equilibrium can be assessed directly using the eigenvalues of the system Jacobian matrix. While this method is considerably faster, it does not provide information of the nonlinear rotor orbit or a possible limit cycle.

In this framework, the main contribution of this paper is to theoretically show that the bump structure compliance has a strong influence on the journal lateral stability, and that the more compliant the bump structure or the heavier the load, the larger the discrepancy between the two approaches becomes. And finally, the possible source of the discrepancy between the two approaches, which is linked to the basic assumptions with regard to the perturbation of the system equations, is discussed.

\section{Mathematical model - time domain}

In Fig 1, the schematics of a perfectly balanced rotor-bearing system is illustrated. With the nodal position vector defined as $\varepsilon=\left\{\varepsilon_{A x}, \varepsilon_{A y}, \varepsilon_{B x}, \varepsilon_{B y}\right\}^{T}$ describing the instantaneous position of the shaft in the bearing locations $A$ and $B$, and under the assumption that the shaft is rigid and subjected to small amplitude vibrations in the $x$ - and $y$-direction, its equations of motion can be written in dimensionless form as

$$
\tilde{\mathbf{M}} \ddot{\boldsymbol{\varepsilon}}-\tilde{\mathbf{G}} \dot{\varepsilon}=\tilde{\mathbf{w}}-\tilde{\mathbf{f}} .
$$

Here, the dimensionless mass and gyroscopic matrices, $\tilde{\mathbf{M}}$ and $\tilde{\mathbf{G}}$, are given in Appendix A, $\tilde{\mathbf{w}}$ is the static load vector and $\tilde{\mathbf{f}}^{T}=\left\{\tilde{\mathbf{f}}_{A}^{T}, \tilde{\mathbf{f}}_{B}^{T}\right\}^{T}$ is the reaction force vector stemming from the bearings. The reaction force vector $\tilde{\mathbf{f}}$ is determined by integration of the fluid film pressure for each bearing as

$$
\tilde{\mathbf{f}}_{\alpha}=\left\{\begin{array}{c}
\tilde{F}_{x} \\
\tilde{F}_{y}
\end{array}\right\}=\int_{0}^{\tilde{L}} \int_{0}^{2 \pi}(\tilde{p}-1)\left\{\begin{array}{c}
\cos (\theta) \\
\sin (\theta)
\end{array}\right\} d \tilde{\theta} d \tilde{z}
$$




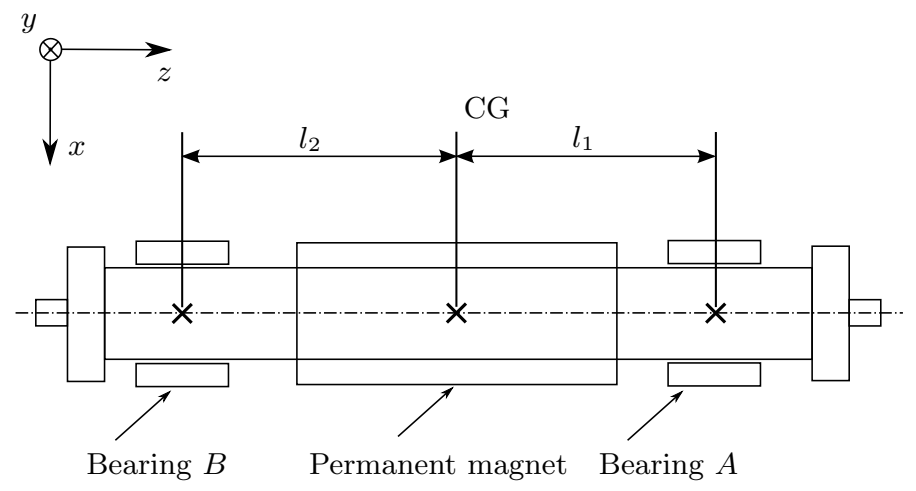

(a) Shaft and bearings.

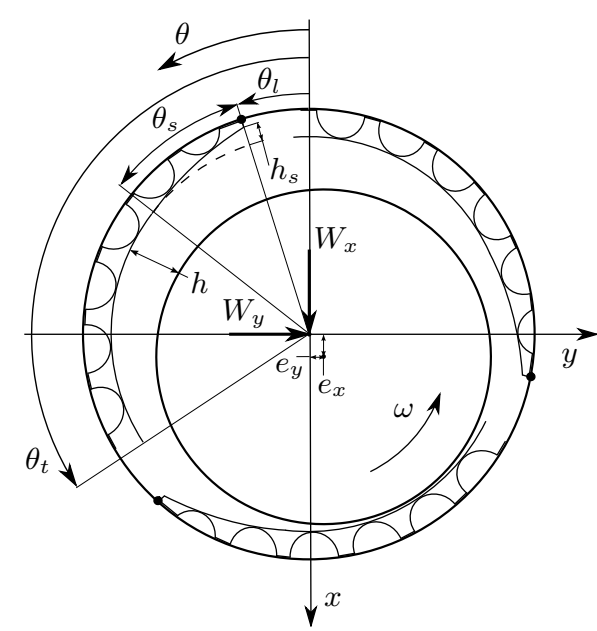

(b) Detailed view of bearing geometry.

Figure 1: Schematics and nomenclature of a rigid rotor supported by foil journal bearings.

where $\alpha=A, B$. The pressure in each bearing can be obtained by solving the Reynolds equation for compressible fluids. This equation can be written in dimensionless vector form [22] as

$$
\nabla \cdot\left(\tilde{p} \tilde{h}^{3} \nabla \tilde{p}\right)=\nabla \cdot(\tilde{p} \tilde{h}) \mathbf{s}+2 S \frac{d}{d \tau}(\tilde{p} \tilde{h})
$$

where the film height is defined as

$$
\tilde{h}\left(\varepsilon_{x}, \varepsilon_{y}, \tilde{\theta}, \tilde{p}, \dot{\tilde{h}}_{c}\right)=\tilde{h}_{r}\left(\varepsilon_{x}, \varepsilon_{y}, \tilde{\theta}\right)+\tilde{h}_{c}\left(\tilde{p}, \dot{\tilde{h}}_{c}\right)
$$

and $\tilde{h}_{r}\left(\varepsilon_{x}, \varepsilon_{y}, \tilde{\theta}\right)$ is the undeformed rigid height, which depends on the rotor eccentricity. For a segmented journal bearing, with inlet slopes and the nomenclature as illustrated in Fig. 1b, the dimensionless rigid film height can be written as

$$
\tilde{h}_{r}\left(\varepsilon_{x}, \varepsilon_{y}, \tilde{\theta}\right)= \begin{cases}1+\varepsilon_{x} \cos (\theta)+\varepsilon_{y} \sin (\theta)-\tilde{h}_{s} \frac{\theta-\theta_{i}}{\theta_{s}}, & \theta_{l i} \leq \theta \leq \theta_{i} \\ 1+\varepsilon_{x} \cos (\theta)+\varepsilon_{y} \sin (\theta), & \theta_{i}<\theta \leq \theta_{t i}\end{cases}
$$

where

$$
\begin{aligned}
\theta_{i} & =\theta_{s}+\theta_{l}+\frac{2 \pi}{N_{p}}(i-1) \\
\theta_{l i} & =\theta_{l}+\frac{2 \pi}{N_{p}}(i-1) \\
\theta_{t i} & =\theta_{t}+\frac{2 \pi}{N_{p}}(i-1) .
\end{aligned}
$$

The pressure dependent deformation of the foils, referred to as the compliant height $\tilde{h}_{c}\left(\tilde{p}, \dot{\tilde{h}}_{c}\right)$, is given in Appendix B based on the assumption of the SEFM [29, 30].

\subsection{Solution strategy}

The equations (1) through (6) constitute the mathematical model for the perfectly balanced rotor-bearing system. In order to simulate the nonlinear rotor response and preserve the true simultaneously coupled nature of the system, a set of ordinary differential equations (ODEs) in the state variables $\varepsilon, \psi, \tilde{h}_{c}$, where $\psi=\tilde{p} \tilde{h}$, is established. This approach was originally suggested by Bonello and Pham [43, 44, 46] and later used by Larsen and Santos [34] for obtaining the nonlinear unbalance response of a rigid rotor similar to the one treated here. In order to obtain the coupled system of ODEs, the Reynolds equation needs to be discretised. 


\subsection{Reynolds equation - discretisation}

The fluid film partial differential equation (PDE) (3) is discretised following a standard Bubnov-Galerkin FE procedure with implementation of an isoparametric element formulation [48]. First, a partial substitution with $\psi$ is performed to obtain

$$
\nabla \cdot\left(\tilde{p} \tilde{h}^{3} \nabla \tilde{p}\right)-\nabla \cdot(\tilde{p} \tilde{h}) \mathbf{s}-2 S \frac{d \psi}{d \tau}=0
$$

which, upon discretisation [49], yields a system of linear equations on the element level

$$
\boldsymbol{\Gamma}^{e} \dot{\boldsymbol{\psi}}^{e}=\mathbf{r}^{e}
$$

where

$$
\boldsymbol{\Gamma}^{e}=2 S \int_{\Phi^{e}} \mathbf{N}^{T} \mathbf{N} d \Phi, \quad \mathbf{r}^{e}=-\int_{\Phi^{e}} \mathbf{B}^{T} \tilde{p} \tilde{h}^{3} \mathbf{B} d \Phi \cdot \tilde{\mathbf{p}}^{e}+\int_{\Phi^{e}} \mathbf{B}^{T} \mathbf{s} \tilde{h} \mathbf{N} d \Phi \cdot \tilde{\mathbf{p}}^{e} .
$$

The element vectors and matrices are expanded to structure size by the usual element summation:

$$
\mathbf{r}=\sum_{e} \mathbf{r}^{e} ; \quad \tilde{\mathbf{p}}=\sum_{e} \tilde{\mathbf{p}}^{e} ; \quad \dot{\psi}=\sum_{e} \dot{\psi}^{e} ; \quad \boldsymbol{\Gamma}=\sum_{e} \boldsymbol{\Gamma}^{e}
$$

where the integrals are numerically integrated using a quadrature rule [48]. The scalar field quantities $\tilde{p}, \tilde{h}$, are calculated in the respective Gauss points $\left(\xi_{i}, \eta_{j}\right)$ by use of the interpolation functions as:

$$
a\left(\xi_{i}, \eta_{j}\right)=\mathbf{N}\left(\xi_{i}, \eta_{j}\right) \mathbf{a}^{e}
$$

where $a$ and $\mathbf{a}^{e}$ are the scalar field quantities and nodal vectors respectively. Note that the right hand side of (8) is denoted $\mathbf{r}^{e}$. In fact, $\mathbf{r}^{e}$ is the residual that needs to be minimised in order to find the static equilibrium of the journal. When integrating the nonlinear equation system in time to assess stability, it is required to start from this static equilibrium position. An efficient method for minimising $\mathbf{r}^{e}$ is given in [45].

\subsection{Coupled system of ODEs}

With the film PDE discretised, the mathematical model can be written as a system of ODEs, on the form $\dot{\mathbf{y}}=$ $\mathbf{g}(\tau, \mathbf{y})$, and solved for all state variables simultaneously. The state-vector is defined as

$$
\mathbf{y}^{T}=\left\{\boldsymbol{\psi}_{A}^{T}, \boldsymbol{\psi}_{B}^{T}, \tilde{\mathbf{h}}_{c A}^{T}, \tilde{\mathbf{h}}_{c B}^{T}, \mathbf{z}_{1}^{T}, \mathbf{z}_{2}^{T}\right\}^{T}
$$

with $\mathbf{z}_{1}=\boldsymbol{\varepsilon}$ and $\mathbf{z}_{2}=\dot{\boldsymbol{\varepsilon}}$. For the particular case of a rigid rotor supported by two bearings, the system dimension of the coupled ODEs are $4 N+8$ with $N$ being the number of nodes in the fluid film FE model of each individual bearing. The system of ODEs takes the form:

$$
\left\{\begin{array}{c}
\dot{\psi}_{A} \\
\dot{\boldsymbol{\psi}}_{B} \\
\dot{\tilde{\mathbf{h}}}_{c A} \\
\tilde{\mathbf{h}}_{c B} \\
\dot{\mathbf{z}}_{1} \\
\dot{\mathbf{z}}_{2}
\end{array}\right\}=\left[\begin{array}{cccc}
\mathbf{0} & \cdots & \mathbf{0} & \mathbf{0} \\
& & & \\
\vdots & \ddots & \vdots & \vdots \\
& & & \mathbf{0} \\
\mathbf{0} & \cdots & \mathbf{0} & \mathbf{I} \\
\mathbf{0} & \cdots & \mathbf{0} & \tilde{\mathbf{M}}^{-1} \tilde{\mathbf{G}}
\end{array}\right]\left\{\begin{array}{c}
\boldsymbol{\psi}_{A} \\
\boldsymbol{\psi}_{B} \\
\tilde{\mathbf{h}}_{c A} \\
\tilde{\mathbf{h}}_{c B} \\
\mathbf{z}_{1} \\
\mathbf{z}_{2}
\end{array}\right\}+\left\{\begin{array}{c}
\mathbf{g}_{\psi_{A}}\left(\boldsymbol{\psi}_{A}, \mathbf{z}_{1}, \mathbf{z}_{2}, \tilde{\mathbf{h}}_{c A}, \dot{\tilde{\mathbf{h}}}_{c A}\right) \\
\mathbf{g}_{\dot{\psi}_{B} B}\left(\boldsymbol{\psi}_{B}, \mathbf{z}_{1}, \mathbf{z}_{2}, \tilde{\mathbf{h}}_{c B}, \dot{\mathbf{\mathbf { h }}}_{c B}\right) \\
\mathbf{g}_{\dot{t}_{c} A}\left(\boldsymbol{\psi}_{A}, \mathbf{z}_{1}, \mathbf{z}_{2}, \tilde{\mathbf{h}}_{c A}\right) \\
\mathbf{g}_{\tilde{h}_{c} B}\left(\boldsymbol{\psi}_{B}, \mathbf{z}_{1}, \mathbf{z}_{2}, \tilde{\mathbf{h}}_{c B}\right) \\
\mathbf{0} \\
\tilde{\mathbf{M}}^{-1}(\tilde{\mathbf{w}}-\tilde{\mathbf{f}})
\end{array}\right\} .
$$

The nonlinear functions on the right hand side are defined as

$$
\mathbf{g}_{\psi \alpha}\left(\psi_{\alpha}, \mathbf{z}_{1}, \mathbf{z}_{2}, \tilde{\mathbf{h}}_{c \alpha}, \dot{\tilde{\mathbf{h}}}_{c \alpha}\right)=\Gamma_{\alpha}^{-1} \mathbf{r}_{\alpha}
$$

and by vectorising the pad deflection given in (B.3)

$$
\mathbf{g}_{\dot{h}_{c} \alpha}\left(\boldsymbol{\psi}_{\alpha}, \mathbf{z}_{1}, \mathbf{z}_{2}, \tilde{\mathbf{h}}_{c \alpha}\right)=\left(\frac{\tilde{\mathbf{p}}_{\alpha}-1}{\tilde{k}_{\alpha}}-\tilde{\mathbf{h}}_{c \alpha}\right) \frac{1}{\eta_{\alpha}}
$$

where $\alpha=A, B$ denotes the individual bearings. In this work, the system of ODEs is solved using the 'CVODE' solver from the Sundials package [50] which is written in C. This solver is based on the backward differentiation formulas and implements an iterative solver procedure for the nonlinear iterations. An efficient program is written in C, and the solution of (8) in each time step is performed using the efficient banded solver DGBSV from the LAPACK library [51]. 


\section{Mathematical model - frequency domain}

In the time domain model, the bearing forces $\mathbf{f}$ are applied on the right hand side (1). Following a perturbation approach, the linearised stiffness and damping of the bearings can be obtained and an equivalent linearised model, with the dynamic contribution from the bearings on the left hand side, can be established as

$$
\tilde{\mathbf{M}} \ddot{\boldsymbol{\varepsilon}}+(\tilde{\mathbf{D}}-\tilde{\mathbf{G}}) \dot{\boldsymbol{\varepsilon}}+\tilde{\mathbf{K}} \boldsymbol{\varepsilon}=\mathbf{0}
$$

Here $\tilde{\mathbf{K}}$ and $\tilde{\mathbf{D}}$ are the dimensionless stiffness and damping matrices containing the linearised bearing coefficients. To obtain these coefficients, a harmonic perturbation method is employed. The method was first suggested by Lund [28] who applied the procedure on rigid gas and oil lubricated bearings. However, the method is commonly used for foil bearings as well [31-33, 35, 36, 38-40, 42]. Assuming that the shaft exhibits small harmonic oscillations around its equilibrium position in the bearing $\left(\varepsilon_{x_{0}}, \varepsilon_{y_{0}}\right)$, the shaft motion can be written as

$$
\varepsilon_{x}=\varepsilon_{x_{0}}+\Delta \varepsilon_{x} \mathrm{e}^{i \tilde{\omega}_{s} \tau} \text { and } \varepsilon_{y}=\varepsilon_{y_{0}}+\Delta \varepsilon_{y} \mathrm{e}^{i \tilde{\omega}_{s} \tau}
$$

where the amplitudes are assumed to be small, that is, $\Delta \varepsilon_{x} \ll 1$ and $\Delta \varepsilon_{y} \ll 1$. This enables a first-order Taylor expansion for the bearing pressure to be obtained as

$$
\tilde{p}=\tilde{p}_{0}+\left(\Delta \varepsilon_{x} \tilde{p}_{x}+\Delta \varepsilon_{y} \tilde{p}_{y}\right) \mathrm{e}^{i \tilde{\omega}_{s} \tau} .
$$

Next, the foil flexibility can be expressed on complex form

$$
\tilde{q}_{c}=\tilde{q} \frac{1-i \eta}{1+\eta^{2}}
$$

and the film height then becomes

$$
\tilde{h}\left(\varepsilon_{x}, \varepsilon_{y}, \tilde{\theta}, \tilde{p}, \eta\right)=\tilde{h}_{r}\left(\varepsilon_{x}, \varepsilon_{y}, \tilde{\theta}\right)+\tilde{q}_{c}(\tilde{p}-1)
$$

where $\tilde{h}_{r}\left(\varepsilon_{x}, \varepsilon_{y}, \tilde{\theta}\right)$ is given by (5) and (6). Substituting (17), (18) and (19) into (7) and (20), discarding second- and higher order terms yields, upon separation of variables, the zeroth- and first-order equations:

$$
\nabla \cdot\left(\tilde{p}_{0} \tilde{h}_{0}^{3} \nabla \tilde{p}_{0}\right)-\nabla \cdot\left(\tilde{p}_{0} \tilde{h}_{0}\right) \mathbf{s}=0
$$

and

$$
\begin{aligned}
\nabla \cdot\left(\tilde{p}_{0} \tilde{h}_{0}^{3} \nabla \tilde{p}_{\gamma}\right)+\nabla \cdot\left(\left(\tilde{h}_{0}^{3}+3 \tilde{h}_{0} \tilde{p}_{0} \tilde{q}_{c}\right) \nabla \tilde{p}_{0} \tilde{p}_{\gamma}\right)-\nabla \cdot\left(\left(\tilde{h}_{0}+\tilde{p}_{0} \tilde{q}_{c}\right) \tilde{p}_{\gamma}\right) \mathbf{s}-2 i \tilde{\omega}_{s}\left(\tilde{h}_{0}+\tilde{p}_{0} \tilde{q}_{c}\right) \tilde{p}_{\gamma} S \\
=-\nabla \cdot\left(3 \tilde{p}_{0} \tilde{h}_{0} f_{\gamma} \nabla \tilde{p}_{0}\right)+\nabla \cdot\left(\tilde{p}_{0} f_{\gamma}\right) \mathbf{s}+2 i \tilde{\omega}_{s}\left(\tilde{p}_{0} f_{\gamma}\right) S
\end{aligned}
$$

where $\gamma=x, y$ and $f_{x}=\cos (\theta)$ and $f_{y}=\sin (\theta)$. The film height at the static equilibrium position is given by

$$
\tilde{h}_{0}\left(\varepsilon_{x_{0}}, \varepsilon_{y_{0}}, \tilde{\theta}, \tilde{p}_{0}, \eta\right)=\tilde{h}_{r}\left(\varepsilon_{x_{0}}, \varepsilon_{y_{0}}, \tilde{\theta}\right)+\tilde{q}_{c}\left(\tilde{p}_{0}-1\right) .
$$

Solving the zeroth-order equation (21) for the static equilibrium eccentricity position $\left(\varepsilon_{x_{0}}, \varepsilon_{y_{0}}\right)$ with $\eta=0$ yields the static film pressure $\tilde{p}_{0}$. This pressure and the associated equilibrium position is then used when solving the first-order equation (22) for the dynamic pressures $\tilde{p}_{x}$ and $\tilde{p}_{y}$. The bearing reaction forces are found by integrating the static pressure $\tilde{p}_{0}$ for each individual bearing over the pad surface

$$
\tilde{\mathbf{w}}_{\alpha}=\left\{\begin{array}{c}
\tilde{W}_{x} \\
\tilde{W}_{y}
\end{array}\right\}=-\int_{0}^{\tilde{L}} \int_{0}^{2 \pi}\left(\tilde{p}_{0}-1\right)\left\{\begin{array}{c}
\cos (\theta) \\
\sin (\theta)
\end{array}\right\} d \tilde{\theta} d \tilde{z}
$$

and the linearised stiffness and damping coefficients are determined following a similar integration procedure of the dynamic pressures $\left(\tilde{p}_{x}, \tilde{p}_{y}\right)$ for each individual bearing as

$$
\tilde{\mathbf{K}}_{\alpha}+i \tilde{\omega}_{s} \tilde{\mathbf{D}}_{\alpha}=\left[\begin{array}{cc}
\tilde{K}_{x x} & \tilde{K}_{x y} \\
\tilde{K}_{y x} & \tilde{K}_{y y}
\end{array}\right]+i \tilde{\omega}_{s}\left[\begin{array}{cc}
\tilde{D}_{x x} & \tilde{D}_{x y} \\
\tilde{D}_{y x} & \tilde{D}_{y y}
\end{array}\right]=\int_{0}^{\tilde{L}_{0}} \int_{0}^{2 \pi}\left[\begin{array}{cc}
\tilde{p}_{x} \cos (\theta) & \tilde{p}_{y} \cos (\theta) \\
\tilde{p}_{x} \sin (\theta) & \tilde{p}_{y} \sin (\theta)
\end{array}\right] d \tilde{\theta} d \tilde{z}
$$


where $\alpha=A, B$ corresponds to the individual bearing locations. Employing a Bubnov-Galerkin FE procedure to discretise and solve the nonlinear zeroth-order equation (21) for a set of eccentricities $\left(\varepsilon_{x}, \varepsilon_{y}\right)$ corresponds to minimising the residual given in (9). To obtain the equilibrium position $\left(\varepsilon_{x_{0}}, \varepsilon_{y_{0}}\right)$, this is then iteratively repeated following a Newton-Raphson approach. The entire procedure is thoroughly explained in [45] together with the discretisation of the first-order equation which leads to a set of linear complex algebraic equations.

With the bearing coefficients in hand, the rotordynamic stability of the linearised model can be evaluated in the frequency domain. First, a state vector $\mathbf{z}=\left\{\mathbf{z}_{1}, \mathbf{z}_{2}\right\}^{T}$ is introduced in order to rewrite the second-order system (16) into a first-order system in the form

$$
\dot{\mathbf{z}}=\mathbf{A z}
$$

where the state matrix is given as

$$
\mathbf{A}=\left[\begin{array}{cc}
\mathbf{0} & \mathbf{I} \\
-\tilde{\mathbf{M}}^{-1} \tilde{\mathbf{K}} & -\tilde{\mathbf{M}}^{-1} \tilde{\mathbf{D}}_{s}
\end{array}\right]
$$

and the stiffness and damping matrices are composed of the local contributions from each bearing

$$
\tilde{\mathbf{K}}=\left[\begin{array}{cc}
\tilde{\mathbf{K}}_{A} & \mathbf{0} \\
\mathbf{0} & \tilde{\mathbf{K}}_{B}
\end{array}\right], \quad \tilde{\mathbf{D}}=\left[\begin{array}{cc}
\tilde{\mathbf{D}}_{A} & \mathbf{0} \\
\mathbf{0} & \tilde{\mathbf{D}}_{B}
\end{array}\right]
$$

and $\tilde{\mathbf{D}}_{s}=\tilde{\mathbf{D}}\left(\tilde{\omega}_{s}\right)-\tilde{\mathbf{G}}$. Both the stiffness and damping matrices $\tilde{\mathbf{K}}\left(\tilde{\omega}_{s}\right)$ and $\tilde{\mathbf{D}}\left(\tilde{\omega}_{s}\right)$ are generally dependent on the angular velocity of the journal and the excitation frequency. By assuming a solution of the form $\tilde{\mathbf{z}}=\tilde{\mathbf{z}}_{0} e^{\lambda \tau}$ and substituting this into (26), the standard eigenvalue problem $\mathbf{A z}=\lambda \mathbf{z}$ can be obtained. Solving this eigenvalue problem yields eight complex eigenvalues that come in complex pairs. The linear stability analysis is basically an evaluation of these complex eigenvalues. If the real part of an eigenvalue is positive, it indicates that the associated eigenmode is unstable.

\section{Boundary conditions}

In dimensionless form, the edge boundary conditions for the bearings, as depicted in Fig. 1b, are:

$$
\begin{aligned}
\tilde{p}\left(\theta_{l}, \tilde{z}\right) & =\tilde{p}\left(\theta_{t}, \tilde{z}\right)=1 \\
\tilde{p}(\theta, \tilde{L} / 2) & =\tilde{p}(\theta,-\tilde{L} / 2)=1 .
\end{aligned}
$$

For the linearised equations (23) and (24), these boundary conditions can be applied straight forwardly. However, with the time domain model, it is necessary to evaluate the film state variable $\dot{\psi}=\dot{\tilde{p}} h+\dot{\tilde{h}} p$. On the pad edges $\tilde{p}=1$ so $\dot{\tilde{p}}=0$. This means that when solving (15), the following boundary conditions must be imposed:

$$
\begin{gathered}
\dot{\psi}\left(\theta_{l}, \tilde{z}\right)=\dot{\psi}\left(\theta_{t}, \tilde{z}\right)=\dot{\tilde{h}}\left(\dot{\varepsilon}_{x}, \dot{\varepsilon}_{y}, \dot{\tilde{h}}_{c}\right) \\
\dot{\psi}(\theta, \tilde{L} / 2)=\dot{\psi}(\theta,-\tilde{L} / 2)=\dot{\tilde{h}}_{\left(\dot{\varepsilon}_{x}, \dot{\varepsilon}_{y}, \dot{\tilde{h}}_{c}\right)}
\end{gathered}
$$

which is achieved by using a standard FE procedure. Symmetry conditions can be exploited in both models by neglecting (29) or (30) on one side respectively, for example on $(\theta, \tilde{L} / 2)$. In this case, the reaction forces need to be multiplied by two. A commonly used boundary condition [45], which is also used in this work, is to assume that the bearing foils deform evenly over the length $\tilde{L}$ of the bearing. This condition is implemented by replacing $\tilde{\mathbf{p}}_{\gamma}$ in (15) by $\tilde{\mathbf{p}}_{\gamma m}$, where $\tilde{\mathbf{p}}_{\gamma m}$ is the arithmetic mean nodal pressure over the length $\tilde{L}$.

In gas bearings, significant sub-ambient pressures may arise. These sub-ambient pressures can cause the top foil to separate from the bumps into a position in which the pressure on both sides of the pad are equalised. Heshmat [30] introduced a set of boundary conditions accounting for this separation effect. However, in this work, a simple Gümbel [22] boundary condition is imposed, which means that sub-ambient pressures are discarded when integrating the pressure (2) and (24) to obtain the bearing force and reaction components, $\left(\tilde{F}_{x}, \tilde{F}_{y}\right)$ and $\left(\tilde{W}_{x}, \tilde{W}_{y}\right)$ respectively, essentially leaving the subambient regions ineffective. This, on the other hand, means that these regions of the bearings need prescribed boundary conditions, $\tilde{p}_{\gamma}=0$ where $\tilde{p}_{0}<1$, when solving the first-order equation (22). Even though the subambient pressures are excluded when calculating the bearing forces and reactions, and in eccense inactivated when calculating the linearised coefficients, it should be emphasised that for both the time- and the frequency-domain solutions, the foil deformation $\tilde{h}_{c}$ are still based on the unmodified pressure. This was chosen to ensure that both models are subjected to completely equivalent boundary conditions. 


\section{Results}

With the nonlinear time domain and the linear frequency domain based methods both defined, a direct comparison between the two in terms of predicting the onset speed of instability (OSI) can be performed. For this comparison, the rotor-bearing system in Fig. 1 with the dimensions and properties as given in Tab. 2 is chosen as the baseline system.

Table 1: Geometry, material properties and operating conditions of the Siemens foil bearing test-rig.

\begin{tabular}{lrlr}
\hline Parameters & Values & Parameters & Values \\
\hline Bearing radius, $R$ & $33.50 \mathrm{~mm}$ & Bump foil height, $h_{b}$ & $0.9 \mathrm{~mm}$ \\
Bearing length, $L$ & $53.00 \mathrm{~mm}$ & Young's modulus of bump foil, $E$ & $2.07 \times 10^{11} \mathrm{~Pa}$ \\
Bearing radial clearance, $C$ & $40 \mu \mathrm{m}$ & Poisson's ratio of bump foil, $v$ & 0.3 \\
Number of pads, $N_{p}$ & 3 & Foil friction coefficient, $\mu_{f}$ & $\approx 0.2$ \\
First pad leading edge, $\theta_{l}$ & $30 \mathrm{deg}$ & Ambient pressure, $p_{a}$ & $1 \times 10^{5} \mathrm{~Pa}$ \\
First pad trailing edge, $\theta_{t}$ & $145 \mathrm{deg}$ & Air viscosity, $\mu$ & $1.95 \times 10^{-5} \mathrm{~Pa} \cdot \mathrm{s}$ \\
Slope extend, $\theta_{s}$ & $30 \mathrm{deg}$ & $l_{1}$ & $201.1 \mathrm{~mm}$ \\
Slope, $h_{s}$ & $50 \mu \mathrm{m}$ & $l_{2}$ & $197.9 \mathrm{~mm}$ \\
Bump foil thickness, $t_{b}$ & $0.127 \mathrm{~mm}$ & $m=m_{x}=m_{y}$ & $21.1166 \mathrm{~kg}$ \\
Top foil thickness, $t_{t}$ & $0.254 \mathrm{~mm}$ & $I_{x x}=I_{y y}$ & $525.166 \cdot 10^{-3} \mathrm{kgm}^{2}$ \\
Bump foil pitch, $S_{b}$ & $7.00 \mathrm{~mm}$ & $I_{z z}$ & $30.079 \cdot 10^{-3} \mathrm{kgm}^{2}$ \\
Bump foil half length, $l_{0}$ & $3.30 \mathrm{~mm}$ & Foil loss factor, $\eta$ & 0.15 \\
\hline
\end{tabular}

This particular system is chosen due to experimental knowledge a priori, with specific regard to the unbalance response at a range of rotor speeds and a variety of unbalance levels and configurations [34]. For the present analysis, the foil structural stiffness is the only parameter to be varied. Three cases are defined with $k=\left\{8.8 \times 10^{16}, 8.8 \times 10^{9}, 4.4 \times\right.$ $\left.10^{9}\right\} \mathrm{N} / \mathrm{m}^{3}$. The first case corresponds to a rigid (non-compliant) bearing, the second case corresponds to the physical case as reported previously [34], and finally, in the last case the foil stiffness is assumed half the actual value. Using the time domain method, the OSI can be determined by repeatedly simulating the nonlinear rotor response at increasing rotor speeds while starting each new simulation from its respective static equilibrium position. The stability of the rotor is then evaluated by closely monitoring when a self-excited vibration starts growing. This is illustrated in Fig. 2 for each of the three cases with static vertical equilibrium positions of $0.719,0.741$ and 0.832 , respectively. In order to evaluate the rotor dynamical stability using the frequency domain method, the Logarithmic Decrement (LD) obtained from the complex eigenvalues of (27) is examined. If the LD is negative, the associated eigenmode is unstable. However, since the state-space matrix $\mathbf{A}$ is dependent on both the shaft speed and perturbation frequency, $\omega$ and $\omega_{s}$ respectively, the eigenvalue problem needs to be solved in the range of interest, for example, $\omega=[5 ; 45] \mathrm{kRPM}$ and $\omega_{s}=[5 ; 450] \mathrm{Hz}$. A contour plot of the LD associated to the first eigenmode for the first stiffness case is illustrated in the diagram of Fig. 3. If the eigenfrequency $\omega_{1}$ (imaginary part) is plotted in the same diagram, the linear stability of the associated mode can now be evaluated. That is, at the point where $\omega_{1}$ (black full line) intersects the zero LD-line and enters the area where the LD is negative, the mode will become unstable. In fact, a Hopf bifurcation [52] will occur due to changes in the dynamic system parameters, which effectively are the stiffness and damping coefficients of the bearings. According to the diagram, this happens at 20,583 RPM and the unstable mode is then vibrating with a frequency of $\omega_{1} \approx 95.2 \mathrm{~Hz}$. The vibration is self-excited with an increasing amplitude (according to linear theory). A similar analysis for the last three eigenmodes indicated no instabilities.

Based on a mesh convergence study, an FE mesh with 1,938 nodes is used for the discretisation, with both the linear frequency domain method as well as the nonlinear time domain method. The linear stability maps for the remaining cases are illustrated in Figs. 4 and 5. The OSI for the three different foil stiffness cases are compared for both methods in Tab. 2. Both methods predict increasing OSI the softer the foil structure becomes, and for the first case $k=8.8 \times 10^{16} \mathrm{~N} / \mathrm{m}^{3}$, the two methods predict a similar OSI. For the two remaining cases however, the discrepancies in predicted OSI are significant, indicating that the discrepancies between the methods increase when lowering the foil stiffness. Keeping in mind that the two methods are completely equivalent in terms of governing equations, discretisation and boundary conditions, these discrepancies are not expected. The two methods should, according to standard stability theory, yield the same results in terms of the OSI. 
Table 2: Foil stiffness for the three cases with the associated calculated linear and nonlinear onset speed of instability.

\begin{tabular}{cllll}
\hline Case & $\begin{array}{l}\text { Stiffness, } k \\
\left(\mathrm{~N} / \mathrm{m}^{3}\right)\end{array}$ & $\begin{array}{l}\text { OSI frequency domain } \\
(\text { revolutions per minute) }\end{array}$ & $\begin{array}{l}\text { OSI time domain } \\
(\text { revolutions per minute) }\end{array}$ & $\begin{array}{l}\text { Relative discrepancy } \\
(\%)\end{array}$ \\
\hline 1 & $8.8 \times 10^{16}$ & 20,583 & 19,450 & 5.83 \\
2 & $8.8 \times 10^{9}$ & 37,039 & 28,900 & 28.1 \\
3 & $4.4 \times 10^{9}$ & 43,287 & 31,400 & 37.9 \\
\hline
\end{tabular}

While the linear stability analysis will only be able to predict the OSI and not the actual rotor response, this can be achieved with the nonlinear time domain method. From Fig 2, it is seen that for the first case with a high foil stiffness, the rotor becomes unstable and the vibration grows until a stable limit cycle occurs. For the two other cases, normal and soft foil stiffness, no stable limit cycles are formed. Again, it is seen that the softer the foils, the higher the OSI becomes, but the faster the vibration grows when crossing the OSI. In Fig 6, the time domain results are plotted in two directions to form the rotor orbits at the OSI. It is clearly seen that the unstable mode is a forward precessing mode. In the same figure, the frequency spectra of the two vibration components are illustrated, and as seen, these frequencies compare reasonably well with those found with the linear frequency domain analysis Figs. 3, 4 and 5.

\section{Conclusions \& Future Aspects}

The comparison between the two methods, described in this work, clearly and surprisingly indicates significant discrepancies in the prediction of the OSI. This is an important finding because the linearised frequency analysis is generally considered to be valid in terms of predicting both the linearised bearing stiffness and damping properties as well as the OSI [31-33, 35-42]. That is, the perturbation technique proposed by Lund [28] is generally regarded to be sufficiently accurate. As a consequence, much scientific research is thus focussed on improving the prediction of these linearised stiffness and damping coefficients with special attention to the foil structure and overall geometric details of the bearings. In the analysis presented in this work, one could question the estimated foil stiffness $k$ and loss factor $\eta$, but they are nevertheless the same for both methods, and hence both methods should yield the same results in terms of the OSI. One possible explanation for the discrepancy between the two methods could be related to the perturbation of the rotor according to (17). As mentioned, the perturbed pressure around e.g. $\varepsilon_{x_{0}}$ with a perturbation $\Delta \varepsilon_{x}$ can be written as a first-order Taylor expansion as $\tilde{p}\left(\varepsilon_{x}\right) \approx \tilde{p}\left(\varepsilon_{x_{0}}\right)+\frac{\partial}{\partial \varepsilon_{x}}\left(\tilde{p}\left(\varepsilon_{x_{0}}\right)\right)\left(\varepsilon_{x}-\varepsilon_{x_{0}}\right)$. Note however, that this is by assuming the pressure $\tilde{p}\left(\varepsilon_{x}\right)$ to be dependent on the rotor eccentricity $\varepsilon_{x}$ only. For a foil bearing with a complaint foil structure, the pressure must also depend on the deformation of the foils. Assuming this, the Taylor expanded pressure becomes:

$$
\tilde{p}\left(\varepsilon_{x}, \tilde{h}_{c}\right) \approx \underbrace{\tilde{p}\left(\varepsilon_{x_{0}}, \tilde{h}_{c_{0}}\right)}_{\text {I }}+\underbrace{\frac{\partial}{\partial \varepsilon_{x}}\left(\tilde{p}\left(\varepsilon_{x_{0}}, \tilde{h}_{c_{0}}\right)\right)\left(\varepsilon_{x}-\varepsilon_{x_{0}}\right)}_{\text {II }}+\underbrace{\frac{\partial}{\partial \tilde{h}_{c}}\left(\tilde{p}\left(\varepsilon_{x_{0}}, \tilde{h}_{c_{0}}\right)\right)\left(\tilde{h}_{c}-\tilde{h}_{c_{0}}\right)}_{\text {III }} .
$$

Term I of (31) is the static pressure in the fluid film when the rotor is in its equilibrium position. Term II contains the pressure derivative with respect to the rotor position in the $x$-direction, i.e. $\tilde{p}_{x}$. This complex pressure derivative can, as mentioned, be integrated over the bearing surface to obtain the bearing stiffness and damping according to (25). However, since $\tilde{h}_{c}=\tilde{q}(\tilde{p}-1)$ depends on $\tilde{p}$, which again depends on $\varepsilon_{x}$, term III of (31) will contribute to the bearing stiffness and damping coefficients. Quantifying the significance of this term, with regard to the bearing coefficients without including it in the perturbed solution, is difficult and considered outside the scope of this work. Nevertheless, the comparison between the nonlinear time domain and the frequency domain methods in this present work indicate that it is in fact significant for softer bearing surfaces.

Based on a bearing geometry, previously investigated by several researchers, and with a comparable foil structure stiffness, Hoffmann et. al. [47] found good agreement between the OSI obtained from a linear model and that from a nonlinear time domain model. Using the present time integration code, the $13.2 \mathrm{kRPM}$ OSI found in [47] for a 5 $\mathrm{N}$ load has been reproduced using a uniform foil stiffness of both $3.87 \times 10^{9} \mathrm{~N} / \mathrm{m}^{3}$, similar to that used in [47], and a much higher value of $3.87 \times 10^{18} \mathrm{~N} / \mathrm{m}^{3}$. Considering the heaviest loaded case of $30 \mathrm{~N}$ and with a foil stiffness of 
$3.87 \times 10^{9} \mathrm{~N} / \mathrm{m}^{3}$, an OSI of approx. $20 \mathrm{kRPM}$ is obtained. Having in mind the different foil stiffness distributions, this is still considered reasonably close to the $22.4 \mathrm{kRPM}$ found in [47]. Next, by increasing the foil stiffness to $3.87 \times 10^{18} \mathrm{~N} / \mathrm{m}^{3}$, only a slight reduction of the OSI to approx. $18 \mathrm{kRPM}$ is observed. This indicates that for the load levels assessed in [47], the foil stiffness is of limited importance and hence also that the compliance levels will be too small for term III of (31) to be significant. The industrial Siemens AFB treated in the present paper is larger and more heavily loaded, implying that the foil flexibility becomes critical to the stability limit. This reasoning is in line with the method discrepancies reported in [47], as these are in fact increasing as the load level is raised $(0.5 \%, 1.6 \%$ and $7.2 \%$ for loads of $5 \mathrm{~N}, 15 \mathrm{~N}$ and $30 \mathrm{~N}$, respectively). In this context, a near-perfect agreement in predicted OSI should be expected for case 1 of the present paper. Much effort has gone into making the two applied models equivalent, but they are however discrete and their implementations rather comprehensive, taking into account two segmented three-pad bearings, inlet slopes and a rigid rotor. The relatively complex rotor-bearing setup was chosen due to the availability of experimental data [34], but in terms of the presented comparison it would possibly have been wiser to model a much simpler system, i.e. a mass supported by a single foil bearing with a single $360^{\circ}$ pad. The remaining discrepancy of $5.83 \%$ is hence ascribed to numerics and to subtle model differences. One such difference could be related to the Gümbel boundary condition, which is enforced separately in the zeroth- and first-order equations in the linear case, while only once in the time domain integration.

In the light of the current findings, the frequency domain methods derived from Lund's perturbation, is considered inaccurate for stability analysis of bearings with flexible foil structures and a certain level of loading. In that regard it should be emphasised that when Lund [28] originally introduced his perturbation method it was for a rigid surface bearing. In the future, the influence on the OSI attributed to the term III from (31) could be further investigated based on a simpler system. For example, a single mass supported by a one-pad foil bearing, possibly without the Gümbel boundary condition. Such investigation could potentially lead to the identification of a criterion for the validity of the linear approach. 

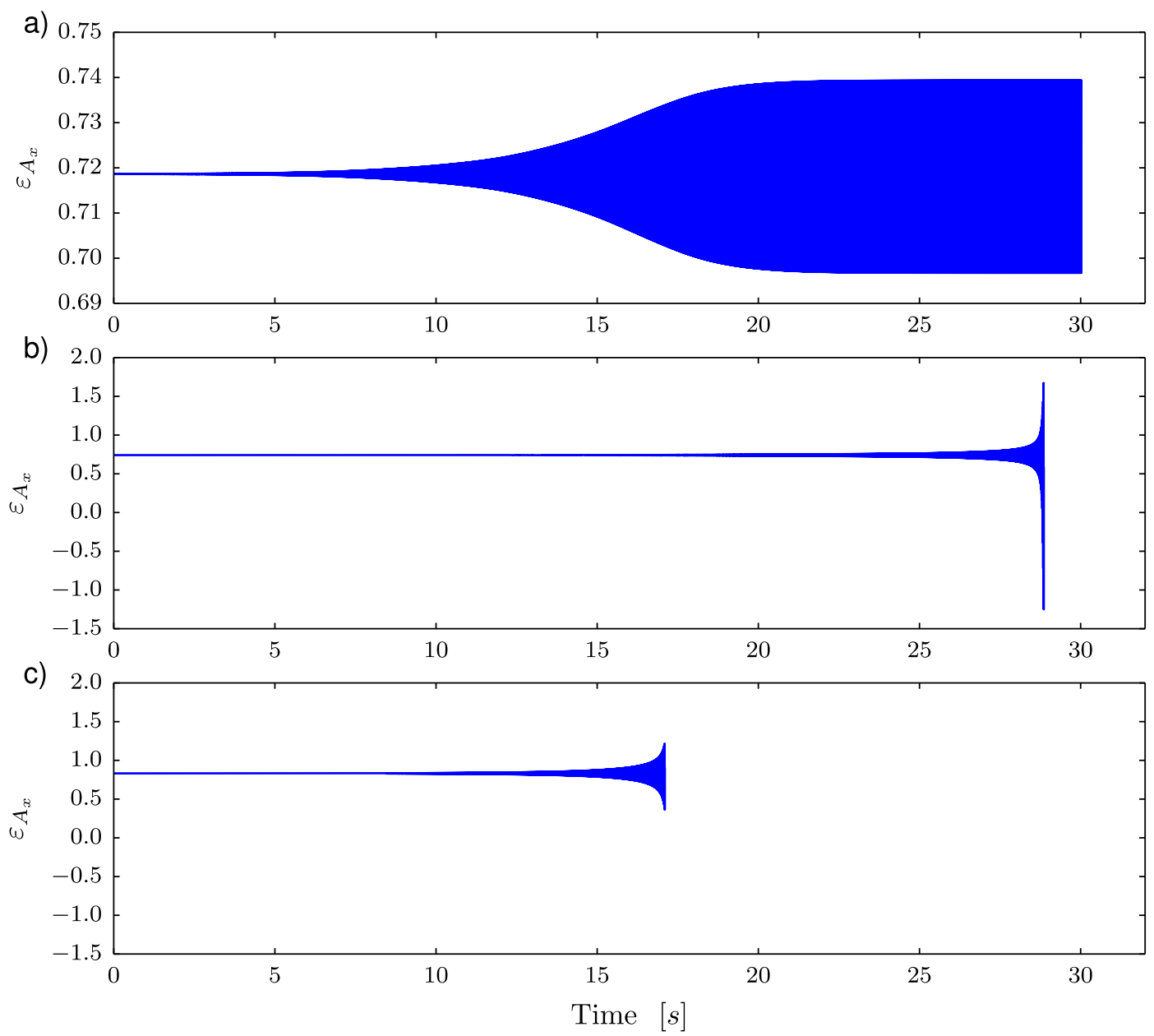

Figure 2: Simulated nonlinear rotor response at the OSI in bearing A vertical direction for three different stiffness values; a) $k=8.8 \times 10^{16} \mathrm{~N} / \mathrm{m}^{3}$, OSI $=19,450 \mathrm{RPM}$ b) $k=8.8 \times 10^{9} \mathrm{~N} / \mathrm{m}^{3}$, OSI $=28,900 \mathrm{RPM}$ and c) $k=4.4 \times 10^{9} \mathrm{~N} / \mathrm{m}^{3}$, OSI $=31,400 \mathrm{RPM}$. The simulations are started from their static equilibrium positions with vertical eccentricities of $0.719,0.741$ and 0.832 , respectively. 


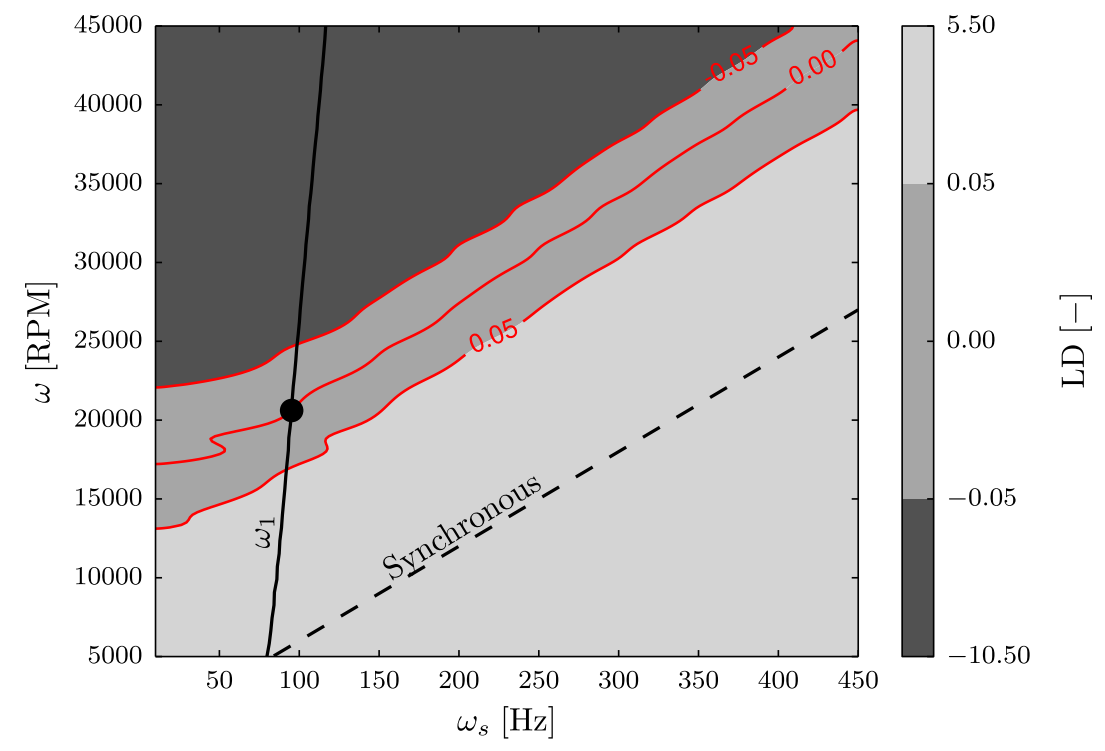

Figure 3: Stability map for case 1 with a foil stiffness of $k=8.8 \times 10^{16} \mathrm{~N} / \mathrm{m}^{3}$. The first eigenmode enters the unstable region at a rotor speed of $\omega=20,583 \mathrm{RPM}$ and the frequency is $\omega_{1}=95.2 \mathrm{~Hz}$. Based on a $50 \times 50\left(\omega_{s}, \omega\right)$ map.

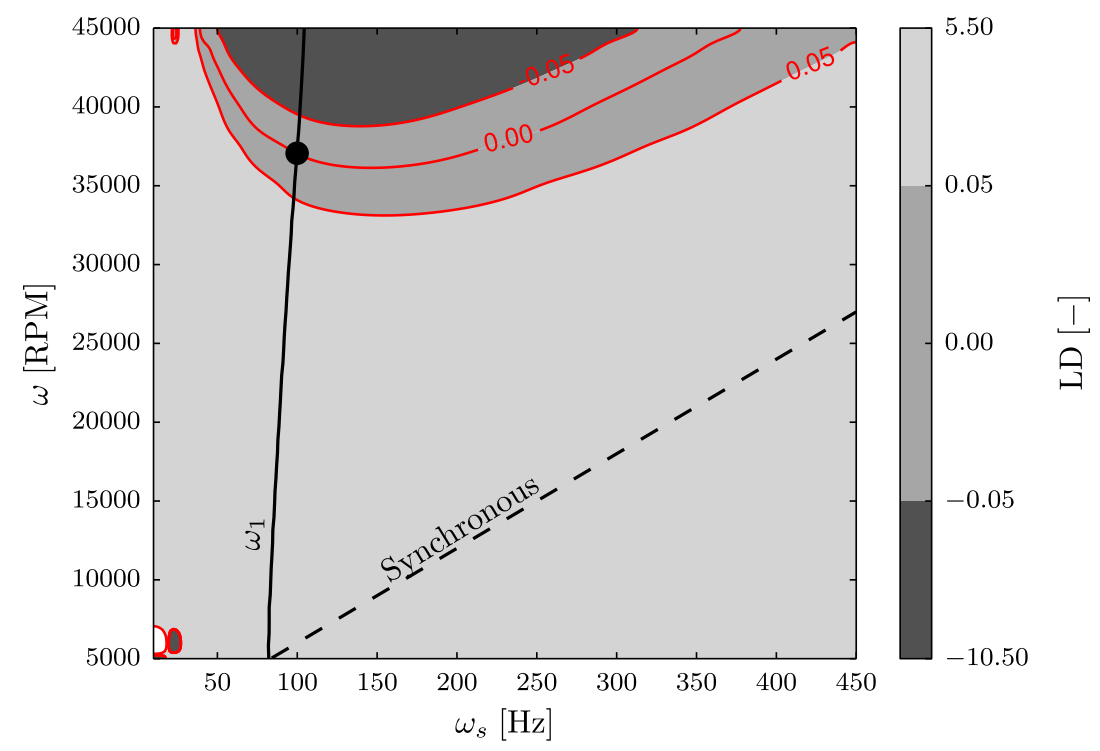

Figure 4: Stability map for case 2 with a foil stiffness of $k=8.8 \times 10^{9} \mathrm{~N} / \mathrm{m}^{3}$. The first eigenmode enters the unstable region at a rotor speed of $\omega=37,039 \mathrm{RPM}$ and the frequency is $\omega_{1}=99.8 \mathrm{~Hz}$. Based on a $50 \times 50\left(\omega_{s}, \omega\right)$ map. 


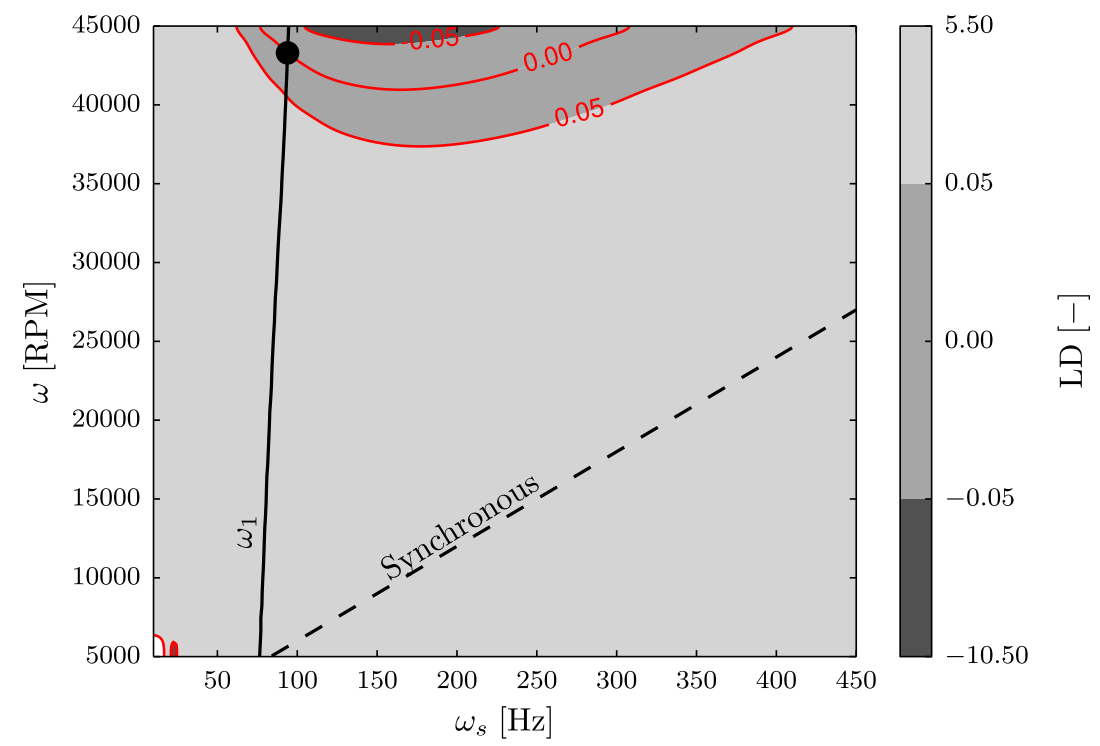

Figure 5: Stability map for case 3 with a foil stiffness of $k=4.4 \times 10^{9} \mathrm{~N} / \mathrm{m}^{3}$. The first eigenmode enters the unstable region at a rotor speed of $\omega=43,287 \mathrm{RPM}$ and the frequency is $\omega_{1}=93.9 \mathrm{~Hz}$. Based on a $50 \times 50\left(\omega_{s}, \omega\right)$ map. 

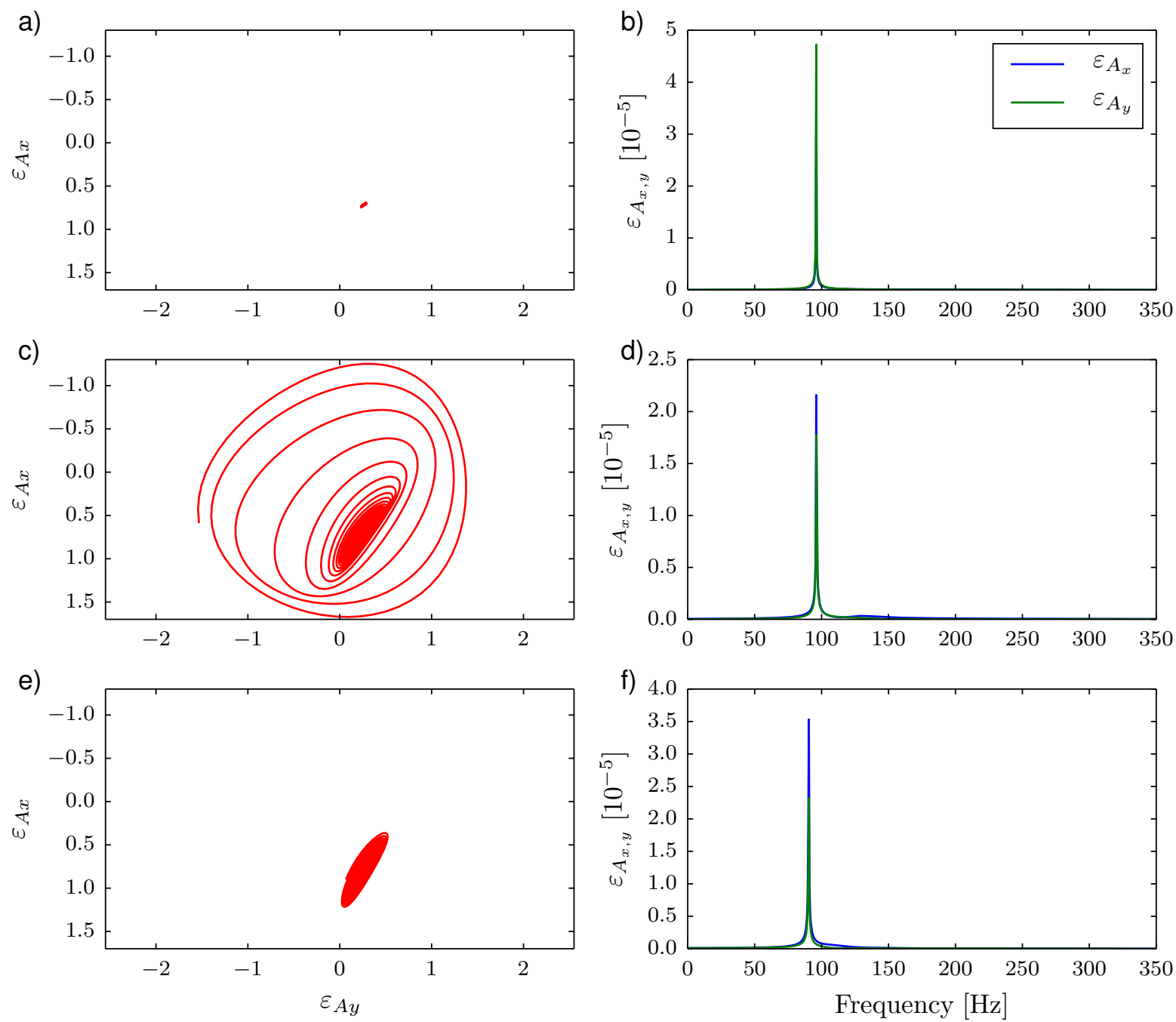

Figure 6: Rotor orbits and frequency spectra obtained with the nonlinear time domain method for the three cases of foil stiffness. The frequency spectra are all based on the first two seconds of each simulation. All simulations are started from static equilibrium. a) Limit cycle obtained for case 1. b) Frequency spectrum for case 1 with a spike at 95.9 Hz. c) Unstable orbit obtained for case 2 with a spike at 95.9 Hz. d) Frequency spectrum for case 2. e) Unstable orbit obtained for case 3. f) Frequency spectrum for case 3 with a spike at $90.4 \mathrm{~Hz}$. 


\section{Appendix A. Rotor model matrices}

The mass and gyroscopic matrices for a rigid rotor can be written as:

$$
\mathbf{M}=\frac{1}{l^{2}}\left[\begin{array}{cccc}
l_{2}^{2} m_{x}+I_{y y} & 0 & l_{1} l_{2} m_{x}-I_{y y} & 0 \\
0 & l_{2}^{2} m_{y}+I_{x x} & 0 & l_{1} l_{2} m_{y}-I_{x x} \\
l_{1} l_{2} m_{x}-I_{y y} & 0 & l_{1}^{2} m_{x}+I_{y y} & 0 \\
0 & l_{1} l_{2} m_{y}-I_{x x} & 0 & l_{1}^{2} m_{y}+I_{x x}
\end{array}\right], \quad \mathbf{G}=\frac{1}{l^{2}}\left[\begin{array}{cccc}
0 & -I_{z z} & 0 & I_{z z} \\
I_{z z} & 0 & -I_{z z} & 0 \\
0 & I_{z z} & 0 & -I_{z z} \\
-I_{z z} & 0 & I_{z z} & 0
\end{array}\right]
$$

The nondimensional form of the mass and gyroscopic matrices are:

$$
\tilde{\mathbf{G}}=\frac{\omega^{2} C}{p_{a} R^{2}} \mathbf{G}, \quad \tilde{\mathbf{M}}=\frac{\omega^{2} C}{p_{a} R^{2}} \mathbf{M} .
$$

\section{Appendix B. Film height}

Discarding the mass of the foil structure, the equation of motion for the foil structure is:

$$
\tilde{p}-1=\tilde{k} \tilde{h}_{c}+\tilde{b} \dot{\hat{h}}_{c} \quad \text { or } \quad \tilde{h}_{c}\left(\tilde{p}, \dot{\tilde{h}}_{c}\right)=\frac{\tilde{p}-1-\tilde{b} \dot{\tilde{h}}_{c}}{\tilde{k}}
$$

Introducing the mechanical loss factor

$$
\eta=\frac{b \omega_{s}}{k}=\frac{\tilde{b}}{\tilde{k}} \frac{\omega_{s}}{\omega}
$$

Inserting the loss factor into the foil equation of motion yields the first-order ordinary differential equation:

$$
\dot{\tilde{h}}_{c}=\left(\frac{\tilde{p}-1}{\tilde{k}}-\tilde{h}_{c}\right) \frac{1}{\eta}
$$

with $\omega_{s} / \omega=1$, meaning that the vibrations in the foil are assumed synchronous.

\section{References}

[1] W. M. Rankine, On the centrifugal force of rotating shafts, Engineer 27 (1869) 249.

[2] S. Dunkerley, On the whirling and vibration of shafts, Philosophical Transactions of the Royal Society of London. A (1894) $279-360$.

[3] H. H. Jeffcott, The lateral vibration of loaded shafts in the neighbourhood of a whirling speed.- the effect of want of balance, The London, Edinburgh, and Dublin Philosophical Magazine and Journal of Science 37 (1919) 304-314

[4] E. Hoextermann, History of steam turbines - 1883 to 1983, 1983.

[5] B. L. Newkirk, H. D. Taylor, Shaft whipping due to oil action in journal bearings, General Electric Review 28 (1925) 559-568.

[6] A. Stodola, Steam and Gas Turbines Vols 1 and 2, Peter Smith, 1945.

[7] B. L. Newkirk, Whirling balanced shafts, in: Proceedings of the Third International Congress on Applied Mechanics, 1930, pp. 105-110.

[8] H. W. Swift, Fluctuating loads in sleeve bearings., Journal of the ICE 5 (1937) 161-195.

[9] D. M. Smith, The motion of a rotor carried by a flexible shaft in flexible bearings, Proceedings of the Royal Society of London. Series A, Containing Papers of a Mathematical and Physical Character (1933) 92-118.

[10] A. C. Hagg, The influence of oil-film journal bearings on the stability of rotating machines, JOURNAL OF APPLIED MECHANICSTRANSACTIONS OF THE ASME 13 (1946) -211.

[11] A. C. Hagg, P. C. Warner, Oil whip of flexible rotors, Trans. ASME 75 (1953) 1339-1344.

[12] A. C. Hagg, G. O. Sankey, Some dynamic properties of oil-film journal bearings with reference to the unbalance vibration of rotors, ASME J. Appl. Mech 78 (1956) 302-306.

[13] Y. Hori, A theory of oil whip, ASME J. Appl. Mech 26 (1959) 189-198.

[14] M. A. Prohl, A general method for calculating critical speeds of flexible rotors, Journal of Applied Mechanics-Transactions of the ASME 12 (1945) -142 .

[15] J. W. Lund, F. K. Orcutt, Calculations and experiments on the unbalance response of a flexible rotor, Journal of Manufacturing Science and Engineering 89 (1967) 785-796.

[16] H. D. Nelson, J. M. McVaugh, The dynamics of rotor-bearing systems using finite elements, Journal of Manufacturing Science and Engineering 98 (1976) 593-600.

[17] W. J. Harrison, The hydrodynamical theory of lubrication with special reference to air as a lubricant, Transactions Cambridge Philosophical Society 22 (1913) 34-54. 
[18] B. Sternlicht, R. C. Elwell, Theoretical and experimental analysis of hydrodynamic gas-lubricated journal bearings, American Society of Mechanical Engineers - Papers (1957).

[19] V. N. Constantinescu, On hydrodynamic instability of gas-lubricated journal bearings, Journal of Fluids Engineering 87 (1965) $579-587$.

[20] E. Reinhardt, J. W. Lund, The influence of fluid inertia on the dynamic properties of journal bearings, Journal of Tribology 97 (1975) $159-165$.

[21] D. M. Smith, Journal bearing dynamic characteristics - effect of inertia of lubricant, in: Proceedings of the Institution of Mechanical Engineers, vol. 179, 1965, pp. 37-44.

[22] B. J. Hamrock, Fundamentals of Fluid Film Lubrication, McGRAW-HILL Series in Mechanical Engineering, McGRAW-HILL, Inc., New York, 1994.

[23] M. J. Braun, W. M. Hannon, Cavitation formation and modelling for fluid film bearings: a review, Proceedings of the Institution of Mechanical Engineers, Part J: Journal of Engineering Tribology 224 (2010) 839-863.

[24] H. G. Elrod, C. W. Ng, A theory for turbulent fluid films and its application to bearings, Journal of Tribology 89 (1967) $346-362$.

[25] J. S. Ausman, An improved analytical solution for self-acting, gas-lubricated journal bearings of finite length, Journal of Basic Engineering 83 (1961) 188-192.

[26] V. Stingelin, Theoretische und experimentelle Untersuchungen an Gaslagern, Ph.D. thesis, Eidgenössischen Technischen Hochschule in Zürich, 1963

[27] J. W. Powell, A review of progress in gas lubrication, Review of Physics and Technology 1 (1970) 96.

[28] J. W. Lund, Calculation of stiffness and damping properties of gas bearings, Journal of Lubrication Technology (1968) 793-804.

[29] H. Heshmat, J. A. Walowit, O. Pinkus, Analysis of gas lubricated compliant thrust bearings, Journal of Lubrication Technology 105 (1983) 638-646.

[30] H. Heshmat, J. A. Walowit, O. Pinkus, Analysis of gas-lubricated foil journal bearings, Journal of Lubrication Technology 105 (1983) 647-655.

[31] D. Kim, Parametric studies on static and dynamic performance of air foil bearings with different top foil geometries and bump stiffness distributions, Journal of Tribology 129 (2007) 354-364.

[32] T. H. Kim, L. San Andrés, Analysis of advanced gas foil bearings with piecewise linear elastic supports, Tribology International 40 (2007) 1239-1245.

[33] T. H. Kim, L. San Andrés, Limits for high-speed operation of gas foil bearings, Journal of Tribology 128 (2006) 670-673.

[34] J. S. Larsen, I. F. Santos, On the nonlinear steady-state response of rigid rotors supported by air foil bearings-theory and experiments, Journal of Sound and Vibration 346 (2015) 284-297.

[35] P. Vleugels, T. Waumans, J. Peirs, F. Al-Bender, D. Reynaerts, High-speed bearings for micro gas turbines: stability analysis of foil bearings, Journal of Micromechanics and Microengineering 16 (2006) 282-289.

[36] R. Hoffmann, T. Pronobis, R. Liebich, The impact of modified corrugated bump structures on the rotor dynamic performance of gas foil bearings, in: Proceedings of ASME Turbo Expo, 2014, pp. -07.

[37] R. Hoffmann, T. Pronobis, R. Liebich, A numerical performance analysis of a gas foil bearing including structural modifications by applying metal shims, in: Proceedings of the 11. International Conference on Schwingungen in Rotierenden Maschinen (SIRM2015), Magdeburg, Germany, 2015

[38] T. H. Kim, L. San Andrés, Heavily loaded gas foil bearings: A model anchored to test data, ASME Conference Proceedings 2005 (2005) $763-771$.

[39] Y. B. Lee, T. H. Kim, C. H. Kim, N. S. Lee, D. H. Choi, Unbalance response of a super-critical rotor supported by foil bearings-comparison with test results, Tribology Transactions 47 (2004) 54-60.

[40] J. P. Peng, M. Carpino, Calculation of stiffness and damping coefficients for elastically supported gas foil bearings, Journal of Tribology 115 (1993) 20-27.

[41] J. S. Larsen, A. J.-T. Hansen, I. F. Santos, Experimental and theoretical analysis of a rigid rotor supported by air foil bearings, Mechanics \& Industry 16 (2015) 106.

[42] J. S. Larsen, I. F. Santos, Compliant foil journal bearings - investigation of dynamic properties, in: Proceedings of 10. International Conference on Schwingungen in Rotierenden Maschinen (SIRM2013), Berlin, Germany, 25-27 February, 2013, pp. 1-12, ISBN 978-3-00-038602-2.

[43] P. Bonello, H. M. Pham, The efficient computation of the nonlinear dynamic response of a foil-air bearing rotor system, Journal of Sound and Vibration 333 (2014) 3459-3478.

[44] P. Bonello, H. M. Pham, Nonlinear dynamic analysis of high speed oil-free turbomachinery with focus on stability and self-excited vibration, Journal of Tribology 136 (2014) 041705. GT2014-25176.

[45] J. S. Larsen, I. F. Santos, Efficient solution of the non-linear reynolds equation for compressible fluid using the finite element method, Journal of the Brazilian Society of Mechanical Sciences and Engineering (2014) 1-13.

[46] H. M. Pham, P. Bonello, Efficient techniques for the computation of the nonlinear dynamics of a foil-air bearing rotor system, in: ASME Turbo Expo 2013: Turbine Technical Conference and Exposition, 2013, pp. -07.

[47] R. Hoffmann, T. Pronobis, R. Liebich, Non-linear stability analysis of a modified gas foil bearing structure, in: Proceedings of the 9th IFToMM International Conference on Rotor Dynamics, 2015, pp. 1259-1276. doi:10.1007/978-3-319-06590-8_103.

[48] R. D. Cook, D. S. Malkus, M. E. Plesha, J. W. Witt, Concepts and applications of finite element analysis, 4 ed., John Wiley, New York, 2002.

[49] J. S. Larsen, B. B. Nielsen, I. F. Santos, On the numerical simulation of nonlinear transient behavior of compliant air foil bearings, in: Proceedings of the 11. International Conference on Vibrations in Rotating Machines, Magdeburg, Germany, 23-25 February, 2015, pp. 1-13.

[50] A. C. Hindmarsh, P. N. Brown, K. E. Grant, S. L. Lee, R. Serban, D. E. Shumaker, C. S. Woodward, Sundials: Suite of nonlinear and differential/algebraic equation solvers, ACM Transactions on Mathematical Software (TOMS) 31 (2005) $363-396$.

[51] E. Anderson, Z. Bai, C. Bischof, S. Blackford, J. Demmel, J. Dongarra, J. Du Croz, A. Greenbaum, S. Hammarling, A. McKenney, D. Sorensen, LAPACK Users' Guide, third ed., Society for Industrial and Applied Mathematics, Philadelphia, PA, 1999.

[52] J. J. Thomsen, Vibrations and stability: advanced theory, analysis, and tools, Springer, 2003. 